\title{
Antisense Oligonucleotide Therapy for Neurodevelopmental Disorders
}

\author{
Sophie F. Hilla, b Miriam H. Meisler ${ }^{a, b}$

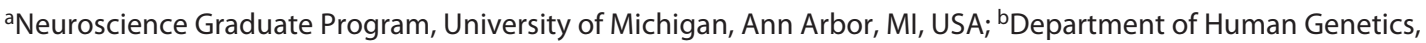 \\ University of Michigan, Ann Arbor, MI, USA
}

\section{Keywords}

Antisense oligonucleotide · Gene therapy · Spinal muscular atrophy · Dravet syndrome $\cdot$ SCN8A

\begin{abstract}
Antisense oligonucleotides (ASOs) are short oligonucleotides that can modify gene expression and mRNA splicing in the nervous system. The FDA has approved ASOs for treatment of ten genetic disorders, with many applications currently in the pipeline. We describe the molecular mechanisms of ASO treatment for four neurodevelopmental and neuromuscular disorders. The ASO nusinersen is a general treatment for mutations of $S M N 1$ in spinal muscular atrophy that corrects the splicing defect in the SMN2 gene. Milasen is a patient-specific ASO that rescues splicing of CNL7 in Batten's disease. STK-001 is an ASO that increases expression of the sodium channel gene SCN1A by exclusion of a poison exon. An ASO that reduces the abundance of the SCN8A mRNA is therapeutic in mouse models of developmental and epileptic encephalopathy. These examples demonstrate the variety of mechanisms and range of applications of ASOs for treatment of neurodevelopmental disorders.
\end{abstract}

\section{Introduction}

The difficulties of treating neurodevelopmental disorders include heterogeneous etiology and the requirement that therapeutic agents cross the blood-brain barrier. Early diagnosis and treatment are essential to prevent irreversible changes. Antisense oligonucleotides (ASOs) are emerging as an exciting new approach to these challenging disorders [1].

ASOs are short oligonucleotides, ten to thirty nucleotides in length, that bind cellular RNAs via complementary base pairing and alter pre-mRNA splicing, mRNA stability, transcription, or RNA-protein interaction. The sequence-specificity of ASO binding results in high specificity and a low level of side effects. ASOs can target proteins that have been considered "undruggable" by directly regulating their expression. ASO therapy can be personalized to target patient-specific mutations $[2,3]$. We describe four examples of neurodevelopmental disorders that demonstrate the range of mutational mechanisms that can be corrected by targeted ASOs.

\section{Chemistry of ASOs}

To confer stability in vivo, ASOs are composed of chemically modified nucleotides with resistance to enzymatic degradation (Fig. 1a). In two common modifica-

Correspondence to:

Miriam Meisler, meislerm@ med.umich.edu 


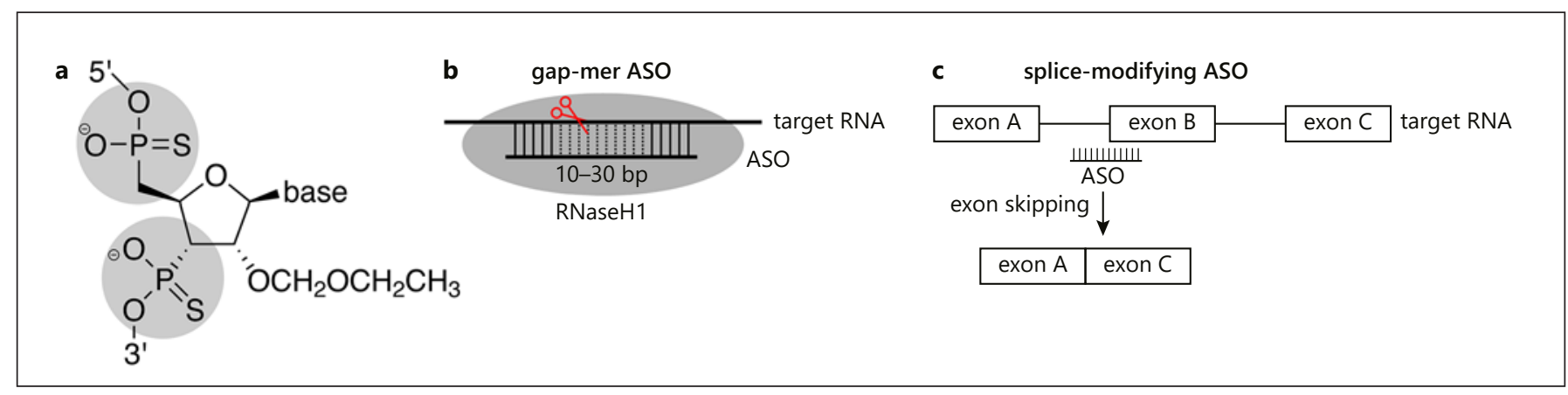

Fig. 1. Chemical modifications of nucleotides in ASOs. To protect ASOs from enzymatic degradation in vivo, modified nucleotides are incorporated. a 2'MOE ribose and phosphorothioate groups. b Gapmer ASOs contain 2'MOE modified bases at their termini (solid lines) and internal unmodified bases (dashed lines) that pro-

tions, a $2^{\prime}$-O-methoxy-ethyl $\left(2^{\prime}-\mathrm{MOE}\right)$ group at ribose position 3 provides resistance to digestion by endonucleases $[4,5]$ while replacement of phosphate along the nucleic acid backbone with phosphorothioate (PS) confers resistance to phosphatases [6]. Other chemical modifications improve binding affinity, solubility, and in vivo stability [7].

Two major types of ASOs are gapmers, which target mRNA for degradation by RNAseH, and ASOs that bind pre-mRNA and alter splicing by steric hindrance. A typical 20-nucleotide gapmer would contain PS bonds throughout and $2^{\prime} \mathrm{MOE}$ modifications at each end (Fig. 1a, b). When the gapmer binds to its target mRNA, the central RNA/DNA hybrid can be cleaved by RNaseH1, resulting in reduced levels of mRNA and protein (Fig. 1b). Since RNaseH1 is active in both the nucleus and cytoplasm, gapmer ASOs can target nuclear RNAs, such as pre-mRNA and long noncoding RNA, as well as mature cytoplasmic mRNA. Gapmers can be used to reduce gene expression as an alternative to RNA interference.

Steric block ASOs contain the 2'MOE and PS modifications throughout and are not substrates for RNaseH. Binding of these ASOs to nuclear pre-mRNA can block interaction with splice factors, resulting in inclusion or exclusion of nearby exons (Fig. 1c).

\section{ASO Therapy for Spinal Muscular Atrophy}

The first neuromuscular disorder successfully treated with an ASO was spinal muscular atrophy (SMA), an autosomal recessive disorder characterized by progressive muscle atrophy [8]. The onset of type I SMA occurs prior to 3 vide a substrate for degradation by RNaseH1. c Binding of steric block ASOs prevents interaction with splice factors, resulting in exon skipping, shown here, or redirection to an alternative splice site. ASOs, antisense oligonucleotides; 2'MOE, 2'-O-methoxyethyl.

months of age and is often fatal by 2 years of age, due to respiratory failure. Affected children have profound muscle weakness and are unable to sit unassisted [9]. Children with type II SMA present between 6 and 18 months of age [9]. Although there is significant mortality, many individuals with type II SMA survive to adulthood [10]. Type III SMA presents later than 18 months of age; patients can sit and walk unaided and have a normal lifespan [9]. The overall frequency of SMA is approximately $1 / 10,000$ [9].

Patients with SMA carry biallelic loss-of-function variants of the SMN1 gene. The survival motor neuron $(\mathrm{SMN})$ protein functions in splicing and assembly of ribonuclear proteins [11]. Human SMN is encoded by 2 nearly-identical genes, $S M N 1$ and $S M N 2$. Most protein is derived from SMN1, due to the presence in the SMN2 gene of a single nucleotide variant in intron 7 that impairs inclusion of exon 7 [12]. As a result, only 15\% of SMN2 transcripts encode full-length protein. The goal of ASO therapy is to increase the inclusion of exon 7 in the SMN2 transcript and restore adequate levels of SMN protein.

The 18-bp ASO nusinersen is complementary to binding sites for splice silencing factors in intron 7 of SMN2 [13] (Fig. 2a). In the presence of this ASO, exon 7 is included in the mRNA and the level of SMN protein is elevated. Preclinical tests in 3 mouse models engineered to express the human SMN2 gene demonstrated an increase in the proportion of SMN2 transcripts containing exon 7 and improvement of SMA phenotypes [14,15]. Intracerebroventricular (ICV) administration in utero improved ear and tail necrosis in a mouse model of SMA type 3 [14]. ICV administration on postnatal day 1 increased body weight, motor performance, and longevity in a model of SMA type 1 [15]. Intrathecal administration to nonhu- 
a SMA, exon inclusion

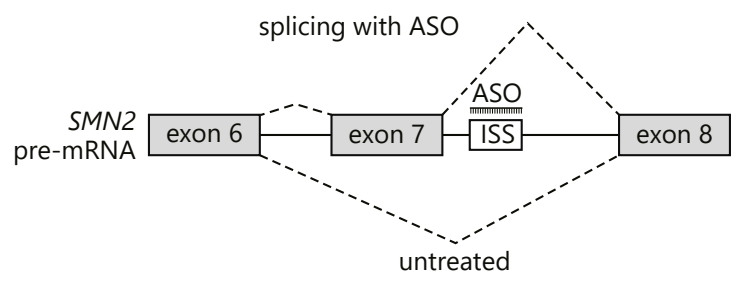

C Dravet syndrome, exclusion of poison exon

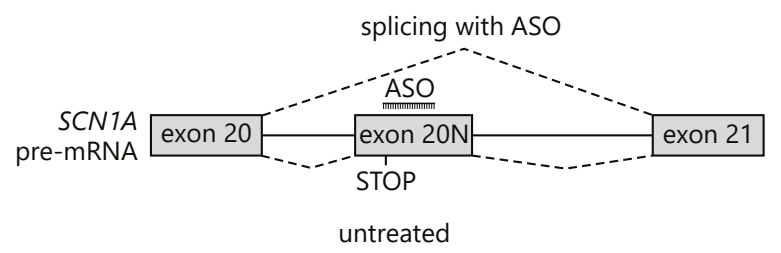

b Batten's disease, exclusion of cryptic exon

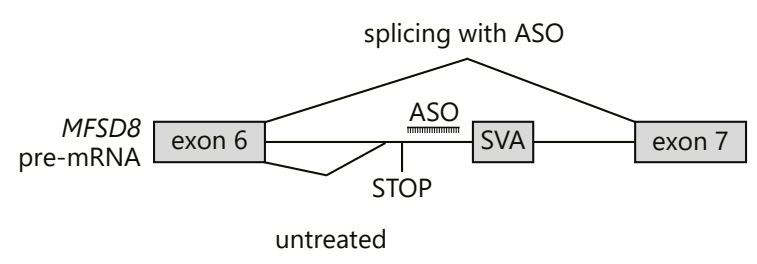

d Scn8a encephalopathy, transcript reduction

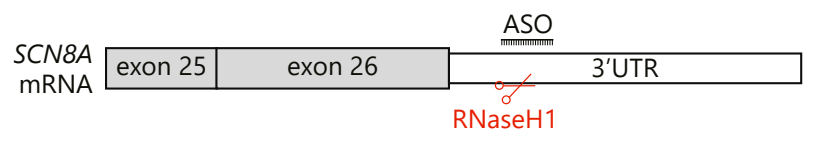

Fig. 2. Mechanisms of ASO therapy for neurodevelopmental disorders. a SMA. The SMN2 gene contains an ISS (intronic splice silencing site) in intron 7. Binding of the ASO to the ISS prevents interaction with splice silencing factors and increases inclusion of exon 7. b Batten's disease. The mutant allele contains an SVA retrotransposon in intron 6 that activates a cryptic splice site. The ASO blocks a splice enhancer, restoring normal splicing. c Dravet syndrome. SCN1A contains the alternative exon $20 \mathrm{~N}$ with an in-

man primates resulted in therapeutic levels of ASO without significant side effects [15].

Nusinersen was approved by the FDA in 2016 for treatment of SMA. This was a major step forward for ASO therapy, establishing the effectiveness of repeated intrathecal delivery of ASOs for treatment of neurological disorders. Children with type I SMA who receive treatment with nusinersen exhibit dramatic improvement. One physician reported that prior to treatment his son was unable to lift his arms or legs and had lost the ability to swallow, but after treatment, he began to lift his head, roll over, and sit on his own, and at the age of 2 years he began to count and learn the alphabet [16]. Children with all 3 types of SMA have been demonstrated to benefit from treatment with nusinersen, with earlier treatment providing greater benefit [17-19].

SMA can also be treated with a recently developed gene replacement therapy, employing the full-length $S M N$ cDNA in an AAV9 adenovirus vector (Zolgensma) [20]. Gene replacement requires only one treatment and is administered by intravenous rather than intrathecal infusion. The long-term outcomes of both treatments require further study.

Antisense Oligonucleotide Therapy for NDDs frame stop codon. Inclusion of exon $20 \mathrm{~N}$ results in protein truncation. The ASO prevents inclusion of exon $20 \mathrm{~N}$ and leads to increased production of full-length SCN1A protein. d SCN8A encephalopathy. Gain-of-function mutations result in elevated neuronal activity. Binding of the ASO to the $3^{\prime} \mathrm{UTR}$ of the mature mRNA provides a substrate for degradation by RNAseH1, leading to reduced protein production. ASO, antisense oligonucleotide; SMA, spinal muscular atrophy.

\section{ASO Therapy for Batten's Disease Type CLN7}

Batten's disease is a group of autosomal recessive lysosomal disorders affecting the central nervous system, also known as neuronal ceroid lipofuscinosis [2]. One type of Batten's disease is caused by mutation of MFSD8 (CLN7), a lysosomal membrane protein. In 2019, an ASO therapy was developed for a patient with a unique mutation of MFSD8 [2]. At the time of diagnosis, the patient was 6 years old and was experiencing developmental regression, ataxia, seizures, brain atrophy, and vision problems.

A retrotransposon insertion into MFSD8 in the mutant allele in this patient generated a cryptic splice site resulting in a transcript with an in-frame stop codon (Fig. 2b). A steric block ASO was designed to bind to a predicted splice enhancer upstream of the SVA retrotransposon in intron 6 (Fig. 2b) [2]. In cultured fibroblasts from the patient, the ASO produced a 3 -fold increase in correctly spliced transcripts and corrected the enlarged lysosomes.

Based on the patient's deterioration and the known clinical course of Batten's disease, the FDA approved an Expanded Access Investigational New Drug application. The treatment regimen was based on the earlier experi-

Dev Neurosci 2021;43:247-252 
ence with nusinersen. The patient received escalating doses of ASO by intrathecal injection, followed by maintenance doses every 3 months. During the first year of treatment, her rapidly worsening symptoms stabilized, but neurological assessment continued to decline, suggesting that treatment may prevent further neurodegeneration but does not restore lost function. Early diagnosis and treatment are critical for treatment of Batten disease.

This ASO, designated Milasen after the patient, is an exciting example of precision therapy for individual patients, so-called " $\mathrm{N}$ of one" therapy. This success demonstrates the importance of developing a standardized process for obtaining FDA approval for individualized ASOs.

\section{ASO Therapy for Dravet Syndrome: A Developmental and Epileptic Encephalopathy}

Developmental and epileptic encephalopathies (DEEs) are rare epilepsy disorders characterized by severe seizures, developmental delay, and intellectual disability. Dravet syndrome is a DEE caused by haploinsufficiency of the sodium channel gene SCN1A. The goal of ASO treatment for Dravet syndrome is to upregulate the wildtype allele, to compensate for the inactive mutant allele. This is possible due to the presence of an alternative "poison" exon in the SCN1A gene with an in-frame stop codon that is included in a subset of SCN1A transcripts [21]. A steric block ASO was designed to prevent inclusion of the poison exon (Fig. 2c). In cultured cells, this ASO reduced the presence of the poison exon from $60 \%$ of transcripts to $<5 \%$ of transcripts [22]. In wild-type mice, administration of this ASO by ICV injection at $\mathrm{P} 2$ resulted in a 3 -fold increase in full-length mRNA and a corresponding increase in SCN1A protein.

Administration of the ASO to haploinsufficient $S c n 1 a$ mice prevented the premature death that occurs in $50 \%$ of untreated mutant mice by 4 weeks of age [22]. ASO treatment also reduced the number of seizures and delayed seizure onset. A phase $1 / 2$ clinical trial of this ASO in Dravet syndrome patients is in progress (https://www. monarchstudy.com/\#!/).

\section{Development of an ASO Treatment for SCN8A Encephalopathy}

SCN8A encephalopathy is a DEE caused by de novo mutations of $S C N 8 A$, which encodes Nav1.6, a major neuronal voltage-gated sodium channel [23]. These mu- tations result in gain-of-function effects on the channel protein, excess neuronal excitability, and seizures [24, 25 ]. To counteract the effects of elevated sodium channel activity, we developed a 20-nucleotide gapmer to reduce the abundance of $S c n 8 a$ transcript via digestion by RNAseH (Fig. 2d), resulting in reduced production of the mutant protein.

Scn 8 a mutant mice were treated with ASO by ICV injection at postnatal day 2, prior to the onset of symptoms [26]. Mice receiving two ASO treatments lived for 9 weeks, compared to the 2 week survival of untreated mice. Three weeks after treatment, Scn 8 a transcript levels were reduced to $50 \%$ of wild-type levels. These results suggest that ASOs reducing expression of $S C N 8 A$ would be therapeutic in humans with $S C N 8 A$ encephalopathy, if the negative consequences of very low expression can be avoided [27].

Haploinsufficiency of SCN1A in Dravet syndrome reduces the firing of inhibitory neurons [28]. We hypothesized that reducing the expression of $\operatorname{Scn} 8 a$ in Dravet syndrome might compensate for the haploinsufficiency of Scnla and restore the excitatory/inhibitory balance. Administration of the Scn8a ASO by ICV injection at postnatal day 2 rescued the $S c n 1 a$ mutant mice, with no deaths or seizures up to 6 months of age [26]. These results suggest that ASO reduction of SCN8A expression could be an effective therapy for patients with additional genetic epilepsies.

\section{Challenges in Development of ASO Therapies}

Several factors complicate the use of ASOs for neurodevelopmental disorders. Since currently constituted ASOs do not cross the blood-brain barrier, injection into the cerebrospinal fluid is required for efficient neuronal uptake. In addition, the limited half-life of ASOs in vivo necessitates 2 to 4 treatments per year. Since both gain-of-function and loss-of-function variants of the same gene can be pathogenic, it is necessary to determine the mode of action of each variant before designing the ASO treatment. The quantitative level of elevated or reduced expression is another important consideration. Treatment of gain-of-function variants of $S C N 8 A$ in DEE requires reduced gene expression, but haploinsufficiency is associated with another, less severe, disorder [26]. Finally, because of the timing of development of the nervous system, it may be necessary to initiate treatment very early. 
Table 1. Status of 4 ASO therapies described in the study

\begin{tabular}{|c|c|c|c|c|}
\hline Gene and disorder & Effect & Chemistry & Status & Reference \\
\hline $\begin{array}{l}\text { SMN2 } \\
\text { SMA (nusinersen) }\end{array}$ & Exon inclusion & Fully modified PS and $2^{\prime}-\mathrm{MOE}$ & FDA approved & {$[13,14,16-18]$} \\
\hline $\begin{array}{l}\text { CLN7 } \\
\text { Batten (Milasen) }\end{array}$ & Exon exclusion & Fully modified PS and $2^{\prime}-\mathrm{MOE}$ & FDA approved & {$[2]$} \\
\hline $\begin{array}{l}\text { SCN1A } \\
\text { Dravet (STK-001) }\end{array}$ & Exon exclusion & Fully modified PS and $2^{\prime}-\mathrm{MOE}$ & Stage 2 clinical trials & {$[20,21]$} \\
\hline $\begin{array}{l}\text { SCN8A } \\
\text { DEE }\end{array}$ & mRNA degradation & Gapmer & Preclinical & {$[25]$} \\
\hline
\end{tabular}

ASO, antisense oligonucleotide; DEE, developmental and epileptic encephalopathy; 2'MOE, 2'-O-methoxyethyl; SMA, spinal muscular atrophy; PS, phosphorothioate.

\section{Conclusion}

The FDA approval of nusinersen in 2016 was a landmark for ASO therapy, demonstrating the feasibility of treating neurological disorders by repeated intrathecal administration of ASOs. The current status of development of the ASOs described here is summarized in $\mathrm{Ta}$ ble 1. Many additional ASOs for treatment of neurodevelopmental disorders are in preclinical development and clinical trials. Improved methods of systemic administration, such as packaging in nanoparticles that cross the blood-brain barrier, would facilitate broader application. Thus far, ASO treatment has been limited to rare genetic diseases. Elucidation of the molecular mechanisms of autism, depression, and Alzheimer's disease may permit the application of ASOs to these common disorders.

\section{Conflict of Interest Statement}

The authors have no conflicts of interest to declare.

\section{Funding Sources}

The preparation of this review was supported by NIH Grant R01 NS0034509-25.

\section{Author Contributions}

The authors contributed equally to preparation of the manuscript.

\section{References}

1 Corey DR. Nusinersen, an antisense oligonucleotide drug for spinal muscular atrophy. Nat Neurosci. Apr 2017;20(4):497-9.

2 Kim J, Hu C, Moufawad El Achkar C, Black LE, Douville J, Larson A, et al. Patient-customized oligonucleotide therapy for a rare genetic disease. N Engl J Med. 2019;381(17): 1644-52.

3 Carroll JB, Warby SC, Southwell AL, Doty $\mathrm{CN}$, Greenlee S, Skotte N, et al. Potent and selective antisense oligonucleotides targeting single-nucleotide polymorphisms in the Huntington disease gene / allele-specific silencing of mutant Huntingtin. Mol Ther. Dec 2011;19(12):2178-85.

4 Monia BP, Lesnik EA, Gonzalez C, Lima WF, McGee D, Guinosso CJ, et al. Evaluation of $2^{\prime}$-modified oligonucleotides containing

Antisense Oligonucleotide Therapy for NDDs
2 -deoxy gaps as antisense inhibitors of gene expression. J Biol Chem. 1993;268(19): 14514-22

5 McKay RA, Miraglia LJ, Cummins LL, Owens SR, Sasmor H, Dean NM. Characterization of a potent and specific class of antisense oligonucleotide inhibitor of human protein kinase C-alpha expression. J Biol Chem. Jan 1999; 274(3):1715-22.

6 Khvorova A, Watts JK. The chemical evolution of oligonucleotide therapies of clinical utility. Nat Biotechnol. Mar 2017;35(3):238-48.

7 Shen W, De Hoyos CL, Migawa MT, Vickers TA, Sun H, Low A, et al. Chemical modification of PS-ASO therapeutics reduces cellular protein-binding and improves the therapeutic index. Nat Biotechnol. Jun 2019;37(6): 640-50.
8 Kolb SJ, Kissel JT. Spinal muscular atrophy: a timely review. Arch Neurol. Aug 2011;68(8): 979

9 Crawford TO, Pardo CA. The neurobiology of childhood spinal muscular atrophy. Neurobiol Dis. Apr 1996;3(2):97-110.

10 Zerres K, Rudnik-Schöneborn S, Forrest E, Lusakowska A, Borkowska J, HausmanowaPetrusewicz I. A collaborative study on the natural history of childhood and juvenile onset proximal spinal muscular atrophy (type II and III SMA): 569 patients. J Neurol Sci. Feb 1997;146(1):67-72.

11 Coovert DD, Le TT, McAndrew PE, Strasswimmer J, Crawford TO, Mendell JR, et al. The survival motor neuron protein in spinal muscular atrophy. Hum Mol Genet. Aug 1997;6(8):1205-14. 
12 Lefebvre S, Bürglen L, Reboullet S, Clermont $\mathrm{O}$, Burlet $\mathrm{P}$, Viollet L, et al. Identification and characterization of a spinal muscular atrophy-determining gene. Cell. Jan 1995;80(1): 155-65.

13 Rigo F, Chun SJ, Norris DA, Hung G, Lee S, Matson J, et al. Pharmacology of a central nervous system delivered 2'-O-methoxyethylmodified survival of motor neuron splicing oligonucleotide in mice and nonhuman primates. J Pharmacol Exp Ther. Jul 2014;350(1): 46-55.

14 Hua Y, Sahashi K, Hung G, Rigo F, Passini $\mathrm{MA}$, Bennett CF, et al. Antisense correction of SMN2 splicing in the CNS rescues necrosis in a type III SMA mouse model. Genes Dev. Aug 2010;24(15):1634-44.

15 Passini MA, Bu J, Richards AM, Kinnecom C, Sardi SP, Stanek LM, et al. Antisense oligonucleotides delivered to the mouse CNS ameliorate symptoms of severe spinal muscular atrophy. Sci Transl Med. Mar 2011;3(72): 72ra18.

16 Hoot NR. "Nusinersen for type 1 spinal muscular atrophy: a father's perspective. Pediatrics. Oct 2019;144(4):e20190226.

17 Chiriboga CA, Swoboda KJ, Darras BT, Iannaccone ST, Montes J, De Vivo DC, et al. Re- sults from a phase 1 study of nusinersen (ISISSMN $\mathrm{Rx}$ ) in children with spinal muscular atrophy. Neurology. Mar 2016;86(10):890-7.

18 Finkel RS, Chiriboga CA, Vajsar J, Day JW Montes J, De Vivo DC, et al. Treatment of infantile-onset spinal muscular atrophy with nusinersen: a phase 2, open-label, dose-escalation study. Lancet. 2016;388(10063):301726.

19 Osredkar D, Jílková M, Butenko T, Loboda T, Golli T, Fuchsová P, et al. Children and young adults with spinal muscular atrophy treated with nusinersen. Eur J Paediatric Neurol. Jan 2021;30(4):1-8.

20 Mendell JR, Al-Zaidy S, Shell R, Arnold WD, Rodino-Klapac LR, Prior TW, et al. Singledose gene-replacement therapy for spinal muscular atrophy. N Engl J Med. Nov 2017; 377(18):1713-22

21 Lim KH, Han Z, Jeon HY, Kach J, Jing E, Weyn-Vanhentenryck S, et al. Antisense oligonucleotide modulation of non-productive alternative splicing upregulates gene expression. Nat Commun. Dec 2020;11(1):3501.

22 Han Z, Chen C, Christiansen A, Ji S, Lin Q, Anumonwo C, et al. Antisense oligonucleotides increase Scnla expression and reduce seizures and SUDEP incidence in a mouse model of Dravet syndrome. Sci Transl Med. Aug 2020;12(558):eaaz6100.

23 Larsen J, Carvill GL, Gardella E, Kluger G, Schmiedel G, Barisic N, et al. The phenotypic spectrum of SCN8A encephalopathy. Neurology. Feb 2015;84(5):480-9.

24 Meisler MH. SCN8A encephalopathy: mechanisms and models. Epilepsia. Dec 2019; 60(S3):S86-91.

25 Meisler MH, Helman G, Hammer MF, Fureman BE, Gaillard WD, Goldin AL, et al. SCN8A encephalopathy: research progress and prospects. Epilepsia. Jul 2016;57(7):1027-35.

26 Lenk GM, Jafar-Nejad P, Hill SF, Huffman LD, Smolen CE, Wagnon JL, et al. Scn8a antisense oligonucleotide is protective in mouse models of SCN8A encephalopathy and Dravet Syndrome. Ann Neurol. 2020;87(3):33946.

27 Meisler MH, Hill SF, Yu W. Sodium channelopathies in neurodevelopmental disorders. Nat Rev Neurosci. 2021;22(3):152-66.

28 Ogiwara I, Miyamoto H, Morita N, Atapour N, Mazaki E, Inoue I, et al. Nav1.1 localizes to axons of Parvalbumin-positive inhibitory interneurons: a circuit basis for epileptic seizures in mice carrying an Scnla gene mutation. J Neurosci. May 2007;27(22):5903-14. 